\title{
Infecciones por enteroparásitos en niños de Sala Cuna y Jardín Infantil de dos Hospitales de Santiago
}

ISABEL NOEMI *, HERNAN REYES * y GABRIELA MAURO **.

Bajo el propósito general de estudiar la frecuencia actual de enteroparásitos en la población del Area de Salud Oriente de Santiago, la Unidad de Parasitología ha efectuado investigaciones en diversos grupos humanos. Uno de ellos es la población infantil, de la cual ya se han comunicado algunos resultados obtenidos en niños atendidos en el Hospital Luis Calvo Mackenna por problemas gastrointestinales (1).

El trabajo actual ccrresponde a investigaciones efectuadas en población infantil no seleccionada desde el punto de vista gastrointestinal, realizadas en el segundo semestre de 1974, en las Salas Cunas y Jardines Infantiles de los Hospitales del Salvador y L. Calvo Mackenna. Con ál se llenaron, además objetivos docentes de la Unidad para el entrenamiento práctico de los estudiantes de Tercer Año de Medicina de nuestra Facultad.

Material y Metodos. El estudio consistió en la investigación de infecciones enteroparasitarias mediante examen microscópico de una muestra fecal por persona y una encuesta epidemiológica adicional.

Las muestras de deposiciones fueron obtenidas en frascos desechables conteniendo alrededor de $5 \mathrm{ml}$ de solución fijadora de formol salino y procesadas por el método de Teleman modificado, según lo consignado en un trabajo anterior (1). Todas las muestras recolectadas fueron primero preparadas y leídas por los estudiantes de medicina y luego por el instructor docente respectivo

\footnotetext{
* Unidad de Parasitologia, Departamento de Medicina Expeperimental. Facultad de Medicína, Sede Santiago Oriente de Ia Universidad de Chile.

** Laboratorio de Parasitología. Hospital Luis Calvo Mackenna, Santlago.

Con la colaboración de los siguientes estudiantes de Tercer Afio de Medicina, 1974.

Orietta Gómez, Hernón De la Barra, Marcelo Valenzuela, Pablo Davanzo, Julio Montt, Susana Urrutla, Nury De la Torre, Patricio Cárdenas, Carlos Farías, Patricio González y John Mackinnon.
}

y el personal técnico de los laboratorios. En el análisis de estos resultados se utilizó la siguiente distribución por edades pediátricas de los niños examinados: Recién nacido (0-30 días), Lactante menor (31 días-1 año), Lactante mayor (1 año 1 día-2 años), Pre-escolar (2 años 1 día-6 años), Escolar (6 años 1 día-14 años). De acuerdo con esta distribución, la población infantil examinada estuvo compuesta por: recién nacidos 0 , lactantes menores 35 , lactantes mayores 42 , preescolares 91 y escolares 3 .

Para la encuesta epidemiológica se procedió a entregar a los padres un formulario especialmente diseñado en el cual se interrogaba sobre antecedentes de diarrea de los niños en los últimos 6 meses, y diversos aspectos de la higiene del hogar (principalmente, calidad del agua de bebida, sistema de eliminación de excretas, existencia de vectores, eliminación de basuras, y número de personas, habitaciones y camas).

\section{T A B L A I}

POSITIVIDAD GENERAL POR PARASITOS Y COMENSALES EN 171 NINOOS EXAMINADOS EN SALA CUNA Y JARDIN INFANTIL DE LOS HOSPITALES SALVADOR Y LUIS CALVO MACKENNA. SANTIAGO, 1974.

\begin{tabular}{lcrc}
\hline $\begin{array}{l}\text { Grupos } \\
\text { de edad }\end{array}$ & Examinados & \multicolumn{2}{c}{ Positivos } \\
& & No & $\%$ \\
\hline R. nacidos & 0 & 0 & 0 \\
L. menores & 35 & 7 & 20,0 \\
L. mayores & 42 & 29 & 68,5 \\
P. escolares & 91 & 54 & 59,3 \\
Escolares & 3 & 1 & 33,3 \\
\hline T o t a l & 171 & 91 & 53,2
\end{tabular}


FRECUENCIA DE INFECCION POR PROTOZOOS INTESTINALES EN 171 NINOS DE SALA CUNA Y JARDIN INFANTIL DE LOS HOSPITALES CALVO MACKENNA Y EL SALVADOR. SANTIAGO, 1974.

\begin{tabular}{|c|c|c|c|c|c|c|c|c|c|}
\hline \multirow{3}{*}{$\begin{array}{l}\text { Grupos } \\
\text { de edad }\end{array}$} & \multicolumn{3}{|c|}{$P a r d s i t o s$} & \multirow{2}{*}{\multicolumn{2}{|c|}{$\begin{array}{c}\text { Entamoeba } \\
\text { histolytica }\end{array}$}} & \multicolumn{3}{|c|}{ Comensales } & \\
\hline & \multirow{2}{*}{ Examinados } & \multicolumn{2}{|c|}{$\begin{array}{l}\text { Giardia } \\
\text { Lamblia }\end{array}$} & & & & eba & \multicolumn{2}{|c|}{$\begin{array}{c}\text { Endolimax } \\
\text { nana }\end{array}$} \\
\hline & & $N^{9}$ & $\%$ & $N^{\circ}$ & $\%$ & $N^{9}$ & $\%$ & No & $\%$ \\
\hline R. nacidos & 0 & 0 & 0 & 0 & 0 & 0 & 0 & 0 & 0 \\
\hline L. menores & 35 & 36 & 17,1 & 0 & 0 & 0 & 0 & 1 & 2,8 \\
\hline L. mayores & 42 & 25 & 59,5 & 0 & 0 & 1 & 2,3 & 2 & 4,7 \\
\hline P. escolares & 91 & 37 & 40,6 & 5 & 5,4 & 5 & 5,4 & 7 & 7,6 \\
\hline Escolares & 3 & 0 & 0 & 0 & 0 & 1 & 33,3 & 0 & 0 \\
\hline $\mathrm{T} \circ \mathrm{t}$ a $\mathrm{I}$ & 171 & 68 & 39,7 & 5 & 2,9 & 7 & 4,09 & 10 & 5,8 \\
\hline
\end{tabular}

T A B L A I I I

RELACION ENTRE ANTECEDENTE DE DIARREA EN LOS ULTIMOS 6 MESES Y PRESENCIA DE G. LAMBLIA EN LAS DEPOSICIONES DE 115 NINOS ENCUESTADOS EPIDEMIOLOGICAMENTE Y POR EXAMEN DE DEPOSICION. SALAS CUNAS Y JARDINES INFANTILES DE LOS HOSPITALES EL SALVADOR Y LUIS CALVO MACKENNA. SANTIAGO, 1974.

\begin{tabular}{lccccc}
\hline & $\begin{array}{c}\text { Con G. lamblia } \\
(45 \text { casos })\end{array}$ & $\begin{array}{c}\text { Sin G. lamblia } \\
(70 \text { casos }) \\
N^{\circ}\end{array}$ & $\begin{array}{c}\text { Total } \\
(115 \\
\text { casos })\end{array}$ & $\begin{array}{c}N^{\circ} \\
\%\end{array}$ & cas \\
\hline Con diarrea & 25 & 55,5 & 32 & 45,7 & 57 \\
Sin diarrea & 20 & 44,5 & 38 & 54,3 & 58 \\
\hline
\end{tabular}

Resultados. El total de niños examinados ascendió a 171 ( 99 hombres y 72 mujeres), de los cuales 100 pertenecían a la S. Cuna y J. Infantil del Hospital del Salvador, y 71 a la del Hospital L. Calvo Mackenna. De estos niños 80 (46,7\%) resultaron negativos al estudio coproparasitológico y $91(53,2 \%)$ estaban infectados por parásitos $\mathrm{y} / \mathrm{o}$ comensales intestinales.

La Tabla I indica la distribución por edad de los niños positivos. Puede observarse que ya en los lactantes menores la cifra de infección general alcanza un $20,0 \%$, que se triplica a $68,5 \%$ en los lactantes mayores y llega a $59,3 \%$ en los preescolares. Dado que en el grupo escolar sólo se estudió a 3 personas, el porcentaje indicado en la tabla tiene relativa validez.

En la Tabla II aparece la distribución de las especies parasitarias $\mathrm{y} / \mathrm{o}$ comensales comprobadas. Aparte de las especies allí indicadas hubo otros casos de infección por comensales que, por su escaso número, no fueron incluídas: 3 casos de infección por Chilomastix mesnili y 1 caso por lodamoeba butschli, todos en lactantes menores. Surgen de esta tabla varios hechos de interés:

a) En el grupo estudiado no se comprobó infección por helmintos intestinales.

b) Giardia lamblia fue el parásito más frecuente, alcanzando una tasa global de $39,7 \%$ y con valores que ascienden desde $17,1 \%$ en lacatntes menores, hasta $59.5 \%$ en lactantes mayores, y bajar luego a $40,6 \%$ en los pre-escolares.

c) La otra especie parasitaria comprobada fue Entamoeba histolytica, con cifras globales de $2,9 \%$ y apareciendo más tardíamente, en el grupo de los pre-escolares, con valores de $5,4 \%$.

d) De los protozoos comensales, Endolimax nana y Entamoeba coli fueron los más frecuentes, con porcentajes totales de $5,8 \%$ y $4,1 \%$, respectivamente. Estas especies comensales sólo tienen importancia en cuanto a la valoración de las condiciones higiénicas de vida en la población encuestada, como un índice complementario de la ingesta de material fecal de origen humano.

La encuesta epidemiológica fue contestada por 115 familias, lo que equivale al $67,2 \%$ de los niños estudiados. De los datos sobre diarrea en los últimos 6 meses hubo 57 respuestas afirmativas y 58 negativas, cuya relación con la presencia o no de $G$. lamblia se indica en la Tabla III. Es interesante destacar que el $55,5 \%$ de los niños con giardiasis tenía antecedentes de diarrea a repetición en los últimos 6 meses y el 44,5\% no lo tenían; en los niños sin giardiasis estos porcentajes se invierten: $45,7 \%$ presentaron diarrea y el $54,3 \%$ no.

En cuanto a la eliminación de excretas, 108 de las 115 familias indicaron disponer de alcantarillado y sólo 7 de pozo séptico. Entre estos úl- 
timos casos la cifra de infección por $G$. lamblia de los niños fue de $70,4 \%$ del grupo respectivo.

El análisis de los restantes aspectos de la encuesta epidemiológica reveló, en general, buenas condiciones higiénicas en el hogar, a juzgar por las respuestas entregadas. Aunque ello nos merece ciertos reparos, no fue posible precisar mejor estos aspectos en la actual oportunidad.

Discusion. Como el estudio fue efectuado sobre la base del examen de una muestra fecal por persona, circunstancia que castiga la positividad global comprobable por exámenes seriados en alrededor del $50 \%(2,3)$, sus resultados tienen valor orientador sobre la realidad parasitológica en el universo examinado.

Esto es especialmente importante con respecto a los altos porcentajes de infección por G. lamblia, que podrían ser mayores si se hubiesen tomado muestras seriadas, en una población infantil no seleccionada por problemas gastrointestinales. Son superiores, incluso, a los comprobados en niños con trastornos digestivos, de edad comparable y procedentes del mismo sector de la ciudad de Santiago, según los resultados de estudios precedentes. Así, Rubio, en 1962 (4), encontró 36,6\% de infección mediante examen de 3 muestras fecales por persona en 270 niños atendidos en el Hospital L. Calvo Mackenna; y Noemí, Reyes y Mauro, en 1974 (1) hallaron un 35,2\%, también con examen de 3 muestras, en 1.000 niños atendidos en el mismo hospital. Son asimismo claramente superiores al 24,5\% determinado por Neghme y Silva, en 1956 (5), para niños de 1-6 años en la zona central de Chile; y al $29,9 \%$ informado por Ramírez y col., en 1972 (6), como tasa general de infección por este parásito en niños de 0-9 años de todo el país. Todo esto nos lleva a afirmar que la infección por $G$. lamblia es un problema enteroparasitario de importancia en los niños atendidos en salas cuna y jardines infantiles, que sería de alto interés estudiar más ampliamente.

Es de suponer que en esos lugares pueden ocurrir infecciones cruzadas entre los niños, dada la estrecha convivencia que allí poseen durante gran parte del día, sumado a los defectos de la higiene de las manos o al mal uso de los servicios higiénicos, tan propios de su corta edad; o a los posibles errores en la manipulación de los alimentos por el personal que los atiende $\mathrm{y}$, tal vez, otros factores favorecedores de la diseminación de la infección.

En estudios previos inéditos y en algunos que están en desarrollo, también en salas cuna y jardines infantiles, hemos comprobado igual fenómeno: G. lamblia aparece como el parásito dominante. Lo mismo nos indican las permanentes consultas a este respecto por los médicos que atienden a estas instituciones. Es por ello que insistimos que la giardiasis es un problema de real importancia en este tipo de población infantil, tanto más cuanto que ya aparece en fuerte proporción en niños de muy poca edad: $17,1 \%$ en los lactantes menores y $59,5 \%$ en los lactantes mayores, según los resultados obtenidos en el presente estudio.

En aparente contraste, las cifras de infección por $E$. histolytica son bastante inferiores, llegando sólo al 2,9\% del total. Debe considerarse, sin embargo, que dicho enteroparásito es el más difícil de demostrar, en especial si se investiga mediante una muestra por persona y con un solo método de examen. Por lo tanto, es posible que en este caso los valores encontrados sean apenas una fracción del problema real.

En cambio, no es extraña la ausencia de infecciones por helmintos enteroparásitos en la población estudiada, dejando expresa constancia que en esta oportunidad no estuvo incluída la investigación de Enterobius vermicularis al no ser utilizado el método adecuado para ello. Respecto de Ascaris lumbricoides y Trichuris trichiura el no haber encontrado infecciones en el grupo infantil estudiado agrega un nuevo argumento a hechos ya observados anteriormente. En efecto, Neghme y Silva en 1963 (7), habían señalado una franca tendencia al descenso de las tasas de infección por estos parásitos en la población infantil de Santiago, atribuyéndolo principalmente a cambios climáticos graduales de la zona central del país que han redundado en menor humedad ambiental, y a la urbanización progresiva de la ciudad, circunstancias ambas que significan seria dificultad para la maduración de los huevos de estos parásitos en el medio exterior y, por ende, reducen sus posibilidades de transmisión. En estudios previos efectuados en población infantil de este mismo sector de la ciudad, Rubio (4) había comprobado 7\% para estos gusanos, y Noemí, Reyes y Mauro (1) constataron tasas inferiores al 1\%. Sin embargo, como ya lo hemos dicho antes (1) esta situación puede sólo reflejar una realidad local, dependiente de la procedencia fundamentalmente urbana de la población infantil examinada.

En relación a Hymenolepis nana, otro helmintos de especial prevalencia en población infantil (8), tampoco fue encontrado en la actual investigación y parece ser también un parásito de baja frecuencia en la población pediátrica del Area de Salud Oriente de Santiago, según estudios previos $(1,4)$.

Considerando los resultados de la encuesta epidemiológica, la relación diarrea-giardiasis fue escogida por ser el parámetro más fácil de objetivar en esa infección según Veghely (9). Al hacerlo así, destaca el hecho que más de la mitad de los niños positivos al parásito había presentado episodios diarreicos recidivantes en los 6 meses ante- 
riores al examen, lo que sugiere que en esos casos $G$. lamblia puede ser un factor primordial en el trastorno. Por otra parte, la cifra de $44,5 \%$ de niños con giardiasis pero sin diarrea, establece una alta proporción de portadores entre estos niños, lo cual constituye un nuevo factor para facilitar las infecciones cruzadas en las salas cuna y jardines infantiles.

\section{RESUMEN}

Mediante el examen de una muestra fecal por persona se estudia la frecuencia de los Hospitales del Salvador y L. Calvo Mackenna, del Area de Salud Oriente de Santiago.

Giardia lamblia es el parásito más frecuente, con una tasa global de $39,7 \%(17.1 \%$ en lactantes menores, $59,5 \%$ en lactantes mayores y $40,6 \%$ en pre-escolares). Entamoeba histolytica ocupa el segundo lugar, con valores generales de 2,9\%. No hubo infecciones por helmintos en la población infantil estudiada, haciendo la salvedad que la infección por Enterobius vermicularis no fue investigada. Considerando tanto la infección por parásitos como la por comensales del intestino, los porcentajes generales son de $53,2 \% \quad 20,0 \%$ en lactantes menores, $68,5 \%$ en lactantes mayores $y$ $59,3 \%$ en pre-escolares).

Más de la mitad de los niños con giardiasis había presentado diarrea a repetición en los 6 meses anteriores al examen.

Se concluye que la giardiasis es un problema parasitario de importancia en niños atendidos en salas cuna y jardines infantiles, que merece ser estudiado en otros sectores de la ciudad y del país.

\section{AGRADECIMIENTOS}

A las Directoras y al personal de las Salas Cuna y Jardines Infantiles de los Hospitales deI Salvador y L. Calvo Mackenna, por la amplia colaboración prestada al desarrollo de este estudio.

\section{REFERENCIAS}

1.-Noemi, I., Reyes, H. y Mauro, C. Enteroparasitosis en la población infantil del Area de Salud Oriente de Santiago. Rev. Chil. Pediatr., 45, 454, 1974

2.-Schenone, H. $y$ col. Encuesta Coproparasitológico en un internado. Significado del distinto número de muestras examinadas. Bol. Chile. Parasit., 28: 15-18, 1973.

3.-Neghme, A., Silva, R. y Artigas, J. El laboratorio en el diagnóstico de la amibiasis intestinal. Bol. Chile. Parasit., 10: 66-71, 1955.

4.-Rubio, M. Encuesta enteroparasitológica en el hospital de niños Luis Calvo Mackenna, Santiago. Consideraciones clínicas y epidemiológicas sobre 270 casos. Bol. Chile. Parasit., 17: 93-99, 1962.

5.- Neghme, A. y Silva, R. Distribución y frecuencia de las enteroparasitosis en Chile. Rev. Serv. Nac. Salud, Chile, 1: 31-154, 1956.

6.-Ramirez, R. y col. Frecuencia en Chile de las infecciones por protozoos $y$ helmintos intestinales (1962-1972). Bol. Chile. Parasit., 27: 116-118, 1972.

7.- Neghme, A. y Silva, R. Estado actual de las infecciones por Ascaria lumbricoides y Trichuris trichiura en la ciudad de Santiago de Chile, Encuestas epidemiológicas. Bol. Chile. Parasit., 18: 54-56, 1963.

8.-Reyes, H., Doren, G. e Inzunza, E. Teniasis humana. Frecuencia actual de la infección por diferentes especies en Santiago de Chile. Bol. Chile. Parasit., 27: 23-29, 1972.

9.-Veghely, J. Giardiasis in Children. Am. J. Dis. Child., 56: 1231-1241, 1938. 